\title{
Présent et futur de l'allergie
}

D'importants progrès ont été accomplis, ces dernières années, concernant les mécanismes de l'allergie. Plusieurs classes de récepteurs des IgE ont été découvertes sur différentes cellules, non seulement les basophiles et les mastocytes, mais aussi les lymphocytes B et des polynucléaires éosinophiles, les plaquettes sanguines, les monocytes et les macrophages. Ces différentes classes de récepteurs et de cellules semblent jouer des rôles spécifiques dans l'inflammation, libérant, par exemple, des médiateurs différents. Parmi ceux-ci, outre les eicosanoïdes, on trouve différentes cytokines qui vont être des éléments clés d'un dialogue dont les partenaires sont aussi bien les cellules effectrices de l'inflammation que les lymphocytes B producteurs d'IgE et les lymphocytes $T$ intervenant dans le processus d'induction et de contrôle de la réponse immune. A ce jour, cependant, les retombées thérapeutiques de toutes ces nouvelles données physiopathologiques restent limitées.

\section{André Capron Jean-Paul Dessaint}

ADRESSE

A. Capron : professeur d'immunologie. J.-P. Dessaint : professeur d'immunologie. Unité mixte, Inserm U.167-Cnrs 624, centre d'immunologie et de biologie parasitaire, Institut Pasteur, 1, rue Calmette, 59019 ongtemps limitées à la description des médiateurs biologiques des composantes immédiates de la réaction d'hypersensibilité, les connaissances de l'allergie se sont, au cours des toutes dernières années, spectaculairement enrichies grâce à l'introduction des outils modernes de la recherche, notamment moléculaires, mais également par la découverte de la diversité des mécanismes cellulaires impliqués dans l'initiation, le déclenchement et la régulation de la réponse allergique. Au plan méthodologique, il est incontestable que l'application intensive des techniques de clonage moléculaire des gènes, de transfection cellulaire et d'expression des protéines recombinantes ont permis la caractérisation structurale et fonctionnelle, à toutes les étapes de la réponse allergique, de molécules impliquées dans la reconnaissance, la signalisation et la communication intercellulaires. Dans le même temps, l'identification de réseaux cellulaires, complexes dans leurs fonctions tant effectrice que régulatrice, concourait à une révision importante des concepts classiques ayant longtemps dominé la compréhension des phénomènes allergiques. Nous nous proposons, par une analyse rapide des axes effecteurs et régulateurs impliqués dans la réponse allergique, d'identifier les promesses de la recherche en allergie de demain, mais aussi les importantes lacunes qui persistent dans notre capacité à utiliser ces connaissances.

\section{Interaction IgE-récepteur}

La base des connaissances de la réponse allergique a longtemps reposé, et repose encore dans l'esprit de beaucoup, sur l'interaction entre une classe d'anticorps (isotype) unique, l'immunoglobuline $\mathrm{E}$ ( $\mathrm{IgE})$, et 
une population cellulaire (mastocytes et basophiles) privilégiée, grâce à sa double capacité - d'exprimer à la membrane des récepteurs de haute affinité pour ce ligand immunoglobulinique - et de pouvoir libérer, à la suite de son activation dépendante des IgE, des médiateurs spécifiques responsables de la réaction inflammatoire.

Le clonage des gènes codant pour la chaîne lourde $\epsilon$ des immunoglobulines a conduit à l'observation d'une conformité surprenante avec les autres immunoglobulines, ce qui contraste avec l'étroite spécificité de la liaison au récepteur. Le gène $\mathrm{C}_{\epsilon}$ est fortement homologue aux autres gènes des chaînes lourdes d'immunoglobulines et témoigne, par ailleurs, d'un haut degré de conservation interspécifique, la seule originalité reposant apparemment sur l'instabilité génétique de la molécule et l'existence d'une séquence switch facilitant la commutation directe $\operatorname{Ig} \mathrm{M}-\mathrm{IgE}$ sans étapes intermédiaires [1].

L'un des acquis les plus significatifs de ces études structurales a été la révision de nos concepts concernant l'interaction IgE-récepteur. Contrairement, en effet, au schéma supposé d'insertion perpendiculaire du Fc (fragment cristallisable, moitiés carboxyterminales des chaînes lourdes $\epsilon$ reliées par un pont disulfure) de l'IgE dans son récepteur, l'interaction se fait par l'une des faces latérales du $\mathrm{FC}_{\mathrm{E}}$. On comprend de ce fait que des peptides monomères modélisés sur la jonction des domaines $\mathrm{C}_{\mathrm{E} 1}-\mathrm{C}_{\mathrm{E} 2}$ et représentant une séquence de 76 aminoacides (positions 301-376) peuvent conduire à mimer structuralement et fonctionnellement l'interaction cellulaire de l'IgE [2, 3] (figure 1).

Dans le même temps, le clonage des gènes codant pour les différentes chaînes du récepteur à haute affinité des $\operatorname{IgE}\left(\mathrm{Fc}_{\mathrm{E}} \mathrm{R}_{\mathrm{I}}\right)$ présent sur les mastocytes basophiles, tout en confirmant la structure tétramétrique, débouchait sur trois notions importantes: la transfection de séquences codant pour seulement deux types de chaînes, $\alpha$ (récepteur proprement dit) et $\gamma$ suffit, au moins chez l'homme, pour faire apparaître la fonction; il existe une très significative analogie de la chaîne $\alpha$ peut s'associer au récepteur de type III pour l'IgG. Cette chaîne $m / s n^{\circ} 10$, vol. 6, décembre 90

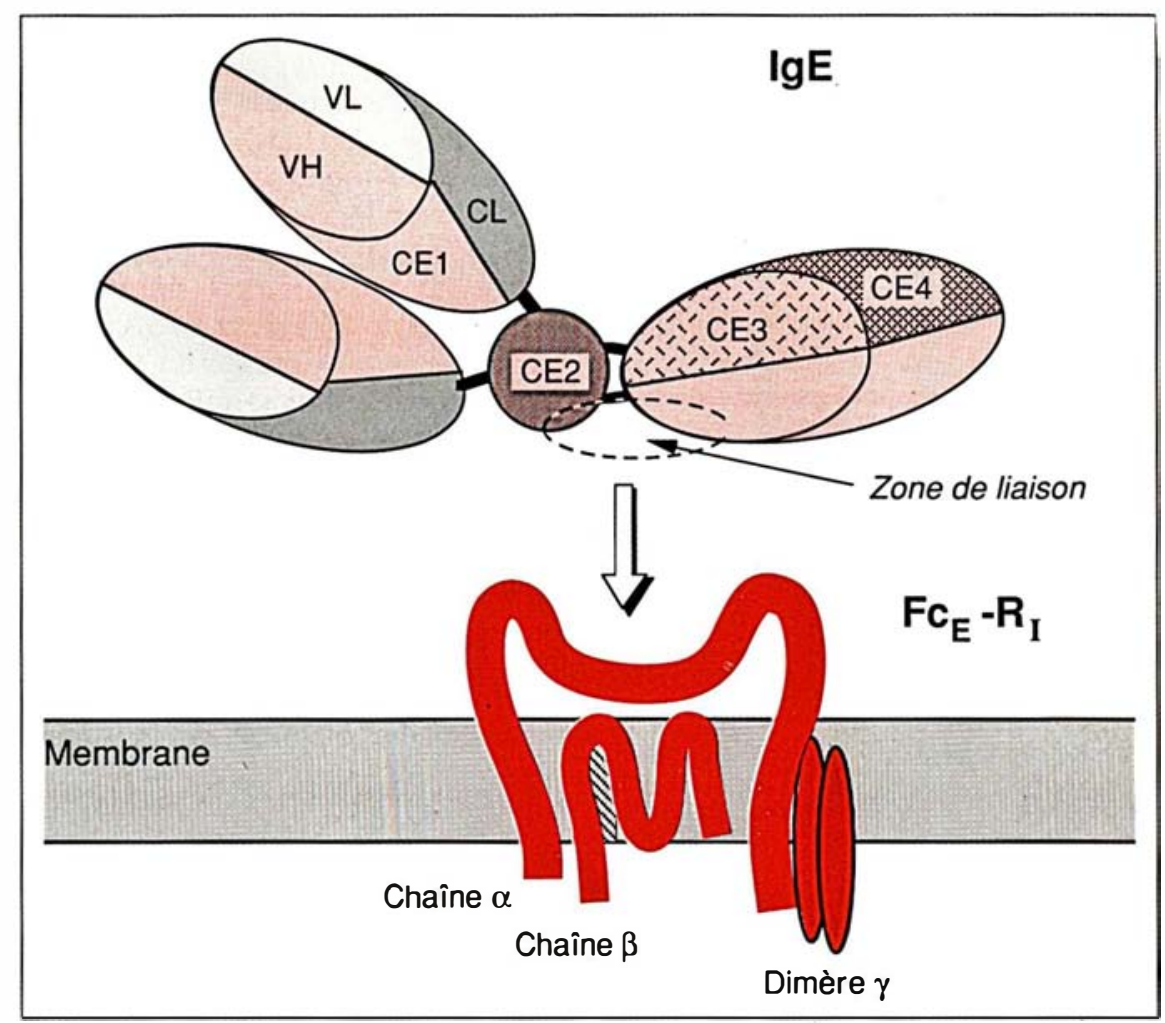

Figure 1. L'interaction de l'IgE avec le récepteur de haute affinité du mastocyte ou du basophile : contrairement au schéma classique, seule la face latérale du $F C_{E}$ se lie à la chaîne $\alpha$ du $F C_{E} R_{1}$. En effet, un peptide de synthèse modélisé sur la région chevauchante des domaines CE1 et CE2 de I'lgE bloque, sous sa forme monomérique, l'interaction de l'immunoglobuline avec son récepteur.

$\gamma$ présente par ailleurs des analogies

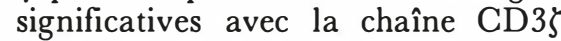
associée au récepteur pour l'antigène des lymphocytes $\mathrm{T}$ [4]. Ces dernières remarques, jointes à la relative banalité de la structure moléculaire de l' $I g E$ elle-même, ne manquent pas de soulever de multiples interrogations sur les raisons qui conduisent à ce qu'une interaction récepteurligand, qui, dans l'état actuel des connaissances, ne possède a priori aucune caractéristique moléculaire remarquable, soit aussi spécifique en termes de reconnaissance réciproque et aussi efficace en termes de fonction.

Ce dialogue privilégié récepteurligand, longtemps confiné au duo mastocytes/basophiles-IgE, s'est en fait, enrichi au cours des dernières années par la mise en évidence de la contribution d'autres partenaires cellulaires. Dans le domaine des cellules effectrices, une série de travaux réalisés dans notre laboratoire a, en effet, permis de révéler l'existence de structures réceptrices pour l'IgE $\left(\mathrm{FC}_{\mathrm{E}} \mathrm{R}_{\mathrm{II}}\right)$ à la membrane de diverses sous-populations cellulaires inflammatoires : monocytes-macrophages, éosinophiles, plaquettes. Ces récepteurs $\mathrm{FC}_{\mathrm{E}} \mathrm{R}_{\mathrm{II}}$ peuvent être considérés comme distincts du premier récepteur $\left(\mathrm{FC}_{\mathrm{E}} \mathrm{R}_{\mathrm{I}}\right)$ sur des bases d'affinité de liaison, d'antigénicité et de structure moléculaire. L'interaction $\operatorname{IgE}-\mathrm{Fc}_{\mathrm{E}} \mathrm{R}_{\mathrm{II}}$ semble conduire, comme dans le cas de mastocytes/basophiles, à des phénomènes d'activation cellulaire permettant aux diverses cellules inflammatoires concernées d'entrer à part entière dans le concert de la réaction allergique [5] (figure 2). 


\section{RÉFÉRENCES}

1. Ishida $\mathrm{N}$, Ueda $\mathrm{S}$, Hayashida $\mathrm{T}$, Honjo $T$. The nucleotide sequence of the mouse immunoglobulin epsilon gene : comparison with the human epsilon gene sequence. EMBO J $1982 ; 1 ; 1117-22$.

2. Padlan EA, Davies DR. A model of the Fc of immunoglobulin E. Mol Immunol $1986 ; 23$ : 1063-75.

3. Helm B, Marsh P, Vercelli D, Padlan E, Gould H, Geha R. The mast cell binding site on human immunoglobulin $\mathrm{E}$. Nature $1988 ; 331$ : 180-3.

4. Miller L, Blanck $\mathrm{U}$, Metzger $\mathrm{H}$ Kinet JP. Expression of high-affinity binding of human immunoglobulin $\mathrm{E}$ by transfected cells. Science 1989 ; 244 : 334-6.

5. Capron A, Dessaint JP, Capron M, Joseph M, Ameisen JC, Tonnel AB. From parasites to allergy : the second receptor for $\operatorname{IgE}\left(\mathrm{Fc}_{\mathrm{E}} \mathrm{R}_{\mathrm{II}}\right)$ Immunol Today 1986; 7 : 15-8.

6. Delespesse G, Sarfati M, Hof stetter H. Human IgE-binding factors. Immunol Today $1989 ; 10: 159-64$.

7. Vercelli D, Helm B, Marsh P, Pad lan E, Geha RS, Gould H. The B cell binding site on human immunoglobulin $\mathrm{E}$. Nature 1989 ; 338 : 649-51.

8. Yokota A, Kikutani H, Tanaka T, et al. Two species of human $\mathrm{Fc}_{\mathrm{E}} \mathrm{R}_{\mathrm{II}}$ (Fc epsilon $\left.\mathrm{R}_{\mathrm{II}} / \mathrm{CD} 23\right)$ : tissue-specific and IL-4 specific regulation of gene expression. Cell 1988 ; $55: 611-8$.

9. Grangette C, Gruart V, Ouaissi MA, et al. $\operatorname{IgE}$ receptor on human eosinophils $\left(\mathrm{Fc}_{\mathrm{E}} \mathrm{R}_{\mathrm{II}}\right)$ : comparison with $\mathrm{B}$ cell $\mathrm{CD} 23$ and association with an adhesion molecule. J Immunol 1989 ; 143 : 3580-8.

10. Capron A, Ameisen JC, Joseph M, Auriault C, Tonnel AB, Caen J. New function for platelets and their pathological implications. Int Archs Allergy Appl Immunol $1985 ; 77$ : 107-14.

11. Stevens RL, Austen KF. Recent advances in the cellular and molecular biology of mast cells. Immunol Today $1989 ; 10: 381-6$.

12. Holgate ST, Hardy C, Robinson C, Agius RM, Howarth PH. The mast cell as a primary effector cell in the pathogenesis of asthma. J Allergy Clin Immunol 1986 ; 77 : 274-82.
On sait que, de manière parallèle, des structures réceptrices pour l'IgE ont été caractérisées - aux plans moléculaire et fonctionnel - au niveau des lymphocytes. Pour ce qui est des lymphocytes B en particulier, une molécule classée comme CD23 paraît à la fois servir de site de liaison spécifique à l'IgE et de récepteur ou messager (après son clivage protéolytique) pour la différenciation des cellules B en producteurs d'anticorps. La connaissance de la structure moléculaire du CD23, grâce au clonage du gène correspondant, a permis de révéler que le $\mathrm{Fc}_{\mathrm{E}}$. $\mathrm{R}_{\mathrm{II}} / \mathrm{CD} 23$ des lymphocytes $\mathrm{B}$ appartient à la famille des lectines animales, donc phylogénétiquement et fonctionnellement distincte de la superfamille des immunoglobulines à laquelle appartient le $\mathrm{Fc}_{\mathrm{E}} \mathrm{R}_{\mathrm{I}}$ [6], et qu'il fixe l'IgE par son domaine lectinique, mais se combine aux chaînes protéiques de l'IgE sur un site du $\mathrm{Fc}_{\mathrm{E}}$ qui n'est que partiellement chevauchant avec le site d'interaction avec le $\mathrm{Fc}_{\mathrm{E}} \mathrm{R}_{\mathrm{I}}$ [7]. L'une des questions essentielles actuellement posée est celle de l'identité entre le $\mathrm{CD} 23 / \mathrm{Fc}_{\mathrm{E}} \mathrm{R}_{\text {II }}$ du lymphocyte $\mathrm{B}$ et les structures réceptrices pour l'IgE des cellules inflammatoires. Des travaux récents ont permis à Kishimoto et al. de distinguer à cet égard deux sousclasses : $\mathrm{FC}_{\mathrm{E}} \mathrm{R}_{\mathrm{II}}$ et $\mathrm{FC}_{\mathrm{F}} \mathrm{R}_{\mathrm{II}}$, qui diffèrent en leur domaine intracytoplasmique et sont engendrées par un épissage alternatif des gènes [8]. Selon le groupe de Kishimoto, $\mathrm{FC}_{\mathrm{E}} \mathrm{R}_{\mathrm{IIa}}$ est exprimé uniquement sur les cellules $\mathrm{B}$ de manière constitutive, cependant que $\mathrm{Fc}_{\mathrm{E}} \mathrm{R}_{\mathrm{IIb}}$ est modulable et s'exprime en particulier sur les cellules B, les macrophages et une lignée éosinophile après induction par l'interleukine 4 (IL-4). En fait, les travaux récents réalisés dans notre laboratoire, par analyse à l'aide d'une batterie d'anticorps monoclonaux anti-CD23 des lymphocytes B et d'un anticorps monoclonal (BB10) préparé vis-à-vis du récepteur pour l'IgE de l'éosinophile humain, semblent indiquer que, en dépit de l'existence de zones d'analogie significative avec le CD23, la partie extracellulaire du $\mathrm{Fc}_{\mathrm{E}} \mathrm{R}_{\mathrm{II}}$ de l'éosinophile pourrait être distincte de celle du lymphocyte B, permettant de supposer que ces diverses structures récep- trices appartiennent à une famille multigénique. Cette étude a aussi révélé l'existence, au sein de la structure du $\mathrm{Fc}_{\mathrm{E}} \mathrm{R}_{\mathrm{II}}$, d'une séquence particulière (adhésiotope), propre à la famille des molécules d'adhérence, dont l'existence apparaît essentielle à la fonction effectrice des cellules inflammatoires [9]. En particulier au niveau des plaquettes, l'expression du $\mathrm{FC}_{\mathrm{E}} \mathrm{R}_{\mathrm{II}}$ semble liée à celle d'une autre molécule d'adhérence, le complexe glycoprotéique $\mathrm{II}_{\mathrm{b}} \mathrm{III}_{\mathrm{a}}$ [10]

Ces différences tenant à l'expression par diverses populations cellulaires de l'une ou l'autre des classes de récepteurs pour l'IgE ne constituent que l'une des facettes de l'hétérogénéité fonctionnelle probable des populations cellulaires concernées. De multiples travaux ont maintenant clairement établi aux plans anatomique et fonctionnel que, au moins dans les modèles animaux, les mastocytes forment deux sous-populations (mastocytes muqueux thymo-dépendants et mastocytes conjonctifs), qui diffèrent par leur aptitude à produire certains médiateurs comme la prostaglandine D2, les leucotriènes peptidiques et le PAF [11].

L'une des conséquences essentielles des mécanismes d'activation cellulaire induite via l'interaction IgE-récepteur est certainement, dans le contexte de l'allergie, la libération - par les cellules concernées - de nombreux médiateurs pouvant rendre compte des manifestations pathologiques observées. Récemment, on a pu démontrer, d'une part, que les mastocytes ou les basophiles ne peuvent plus être considérés comme la seule source cellulaire de ces médiateurs [12] et, d'autre part, qu'au sein même de l'extraordinaire diversité des médiateurs produits existe une certaine hiérarchie, au moins en ce qui concerne leur participation à la phase aiguë de la réaction d'hypersensibilité dépendante des IgE. Les médiateurs lipidiques, et en particulier le PAF-acéther, semblent jouer un rôle majeur. Il est par ailleurs maintenant établi que les sources cellulaires essentielles de la production de cet important médiateur de l'inflammation sont représentées, outre le neutrophile, par les cellules inflammatoires exprimant le $\mathrm{Fc}_{\mathrm{E}} \mathrm{R}_{\mathrm{II}}$ et stimulées par l' $\mathrm{IgE}$ tels l'éosino- 
phile, le macrophage et, dans une moindre mesure, la plaquette [13]. Ces mêmes populations cellulaires peuvent, dans des réactions dépendantes des $\operatorname{IgE}$, libérer non seulement des médiateurs actifs de l'inflammation aiguë, mais aussi des molécules pouvant rendre compte des phénomènes d'agression et de destruction tissulaire. C'est ainsi, par exemple, qu'outre la génération des radicaux libres oxygénés, les macrophages sécrètent de l'interleukine 1 (IL-1) et le tumor necrosis factor/cachectine $(\mathrm{TNF}-\alpha)$, et que les éosinophiles libèrent par un processus de dégranulation sélective leurs composants granulaires: protéine basique majeure (MBP) et peroxydase spécifique (EPO), dont la libération in vivo et le rôle cytotoxique in vitro sont maintenant clairement établis [14]. Ici encore, le clonage des gènes codant pour ces molécules, réalisé très récemment, permet une définition précise de leur structure et l'abord du problème fondamental des rapports structure-fonction.

La notion d'hétérogénéité fonctionnelle déjà évoquée trouve une illustration particulière dans le cas des fonctions effectrices des éosinophiles. Ces cellules forment deux souspopulations caractérisées non seulement par leur différence de densité, d'où la dénomination d'éosinophiles normodenses et hypodenses, mais également par leur capacité d'expression des récepteurs d'IgE $\left(\mathrm{Fc}_{\mathrm{E}} \mathrm{R}_{\mathrm{II}}\right)$, leur sélectivité de réponse à certains ligands ou leur production préférentielle de certains médiateurs, le PAF, par exemple, n'étant synthétisé que par les éosinophiles hypodenses dans des conditions de stimulation dépendante des IgE. Récemment, des arguments fonctionnels et d'ultrastructure ont permis de révéler l'existence, au niveau des éosinophiles, de processus d'exocytose préférentielle, étroitement dépendants de la nature des ligands activateurs. C'est ainsi, par exemple, que la peroxydase de l'éosinophile (EPO) n'est libérée qu'après activation $\operatorname{IgE}$ - ou $\operatorname{IgA}$ dépendante, tandis que la protéine cationique de l'éosinophile (ECP) n'est pas libérée après stimulation via l'IgE et ne l'est qu'après activation IgG- ou IgA-dépendante [15].

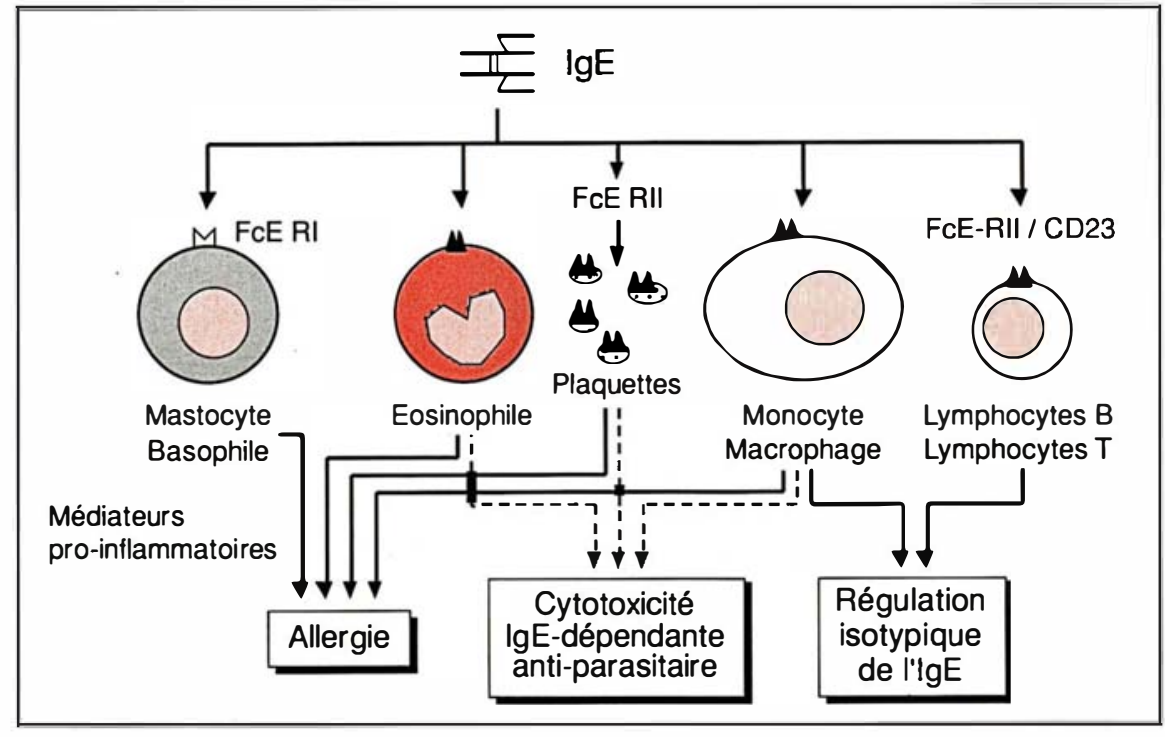

Figure 2. Les deux facettes des fonctions de I'IgE : allergie et immunité antiparasitaire; I'IgE interagit sur diverses populations cellulaires par l'intermédiaire de deux types de récepteurs $\left(F c_{E} R_{1}\right)$ et $\left(F c_{E} R_{\| I}\right)$.

\section{Rôle des interleukines}

L'un des apports les plus originaux des recherches récentes a été la démonstration que des populations cellulaires impliquées dans des fonctions effectrices pouvaient, en même temps qu'elles libéraient des médiateurs pro-inflammatoires, produire des facteurs de régulation de toutes les étapes de la réponse allergique. C'est ainsi que certains mastocytes, dans le contexte de réactions dépendantes des $\operatorname{IgE}$, libèrent une variété d'interleukines presque aussi vaste que celle des lymphocytes $T$ [11], dont certaines (IL-4, IL-5, IL-6) peuvent jouer un rôle essentiel dans le recrutement et l'activation des cellules de l'inflammation, voire la régulation de la production d'anticorps, y compris l'IgE elle-même. De même, en réponse à l'IgE, les monocytes/macrophages libèrent l'IL-1, cofacteur essentiel de l'activation des lymphocytes $\mathrm{T}$ inducteurs qui contrôlent la production d'IgE [15]. Ces observations doivent être replacées dans le contexte général du rôle des interleukines, désormais considéré comme essentiel, à la fois dans la régulation des cellules effectrices et l'induction même de leur différenciation (figure 3).

Chacun peut mesurer la remarquable explosion de connaissances qui, a u cours des dernières années, a accompagné la caractérisation, le clonage, l'expression et l'utilisation expérimentale des diverses interleukines. Le caractère pluripotentiel de nombre de ces interleukines, susceptibles d'agir à la fois sur la croissance et la différenciation d'une même cible cellulaire ou de sous-populations distinctes, leur confère désormais un rôle central dans la compréhension de l'induction et de l'expression des phénomènes allergiques. Deux notions de particulière importance peuvent être dégagées de ce vaste domaine de recherches. La première tient à l'identification, dans les modèles murins, de sous-populations lymphocytaires $\mathrm{T}$ inductrices, connues sous la dénomination de Th1 et Th2, et dont l'équilibre des profils de sécrétion distincts joue un rôle clé dans l'orientation des modalités de la réponse immunitaire. C'est ainsi que, au moins chez la souris, les souspopulations Th1 produisent essentiellement de l'interleukine 2 (IL-2) et de l'interféron- $\gamma$ cependant que les cellules Th2 sont spécifiquement impliquées dans la production de l'interleukine 4 (IL-4) et de l'inter- 
leukine 5 (IL-5). Le fait le plus important réside sans doute dans la démonstration que la production d'IFN- $\gamma$ par les cellules Th1 constitue un facteur essentiel de régulation négative de la production d'IL-4 par les cellules Th2, tandis que les lymphocytes Th2 s'opposent à l'utilisation de l'IL-2 par les cellules Th1. La seconde notion, s'agissant plus particulièrement $\mathrm{du}$ domaine $\mathrm{de}$ l'allergie, est la démonstration : d'une part, que les mastocytes stimulés par l'IgE fonctionnent comme les cellules Th2; d'autre part, qu'au sein de la cascade complexe des interleukines, certaines d'entre elles interviennent de manière privilégiée, soit directement dans l'induction de la réponse $\operatorname{IgE}$, soit dans la différenciation fonctionnelle des cellules effectrices. Le rôle essentiel joué par l'IL-3 et l'IL-4 dans le recrutement et la différenciation des mastocytes thymo-dépendants [11], celui de l'IL-5 dans la différenciation et l'activation des éosinophiles [16], de l'IFN- $\gamma$ dans l'activation des monocytes-macrophages et des plaquettes [15] sont maintenant parfaitement établis. L'action de ces interleukines peut avoir, au niveau des fonctions cellulaires, des effets multiples, dont l'augmentation d'expression des récepteurs membranaires pour l'IgE (IFN- $\gamma$ sur les macrophages et les plaquettes, IL-4 sur les $\mathrm{FC}_{\mathrm{E}} \mathrm{R}_{\mathrm{II}}$ des monocytes-macrophages, IL-5 sur les éosinophiles). Si la réalité de l'existence chez l'homme des sous-populations Th1 et Th2, ou en tout cas d'une distinction fonctionnelle des cellules $\mathrm{T}$ inductrices, reste discutée, il demeure que, au moins in vitro, le rôle des interleukines concernées paraît identique à celui démontré dans les modèles animaux. L'activité des interleukines déborde par ailleurs largement le cadre de la fonction des cellules effectrices et s'exerce de manière décisive lors des événements initiaux conduisant à l'induction de la production d'IgE. S'il est encore difficile de situer dans ce contexte la part exacte jouée par l'IL-4 vis-à-vis des formes solubles des récepteurs de classe II pour l'IgE (IgE binding factors), certaines observations récentes permettent de penser que la commutation isotypique

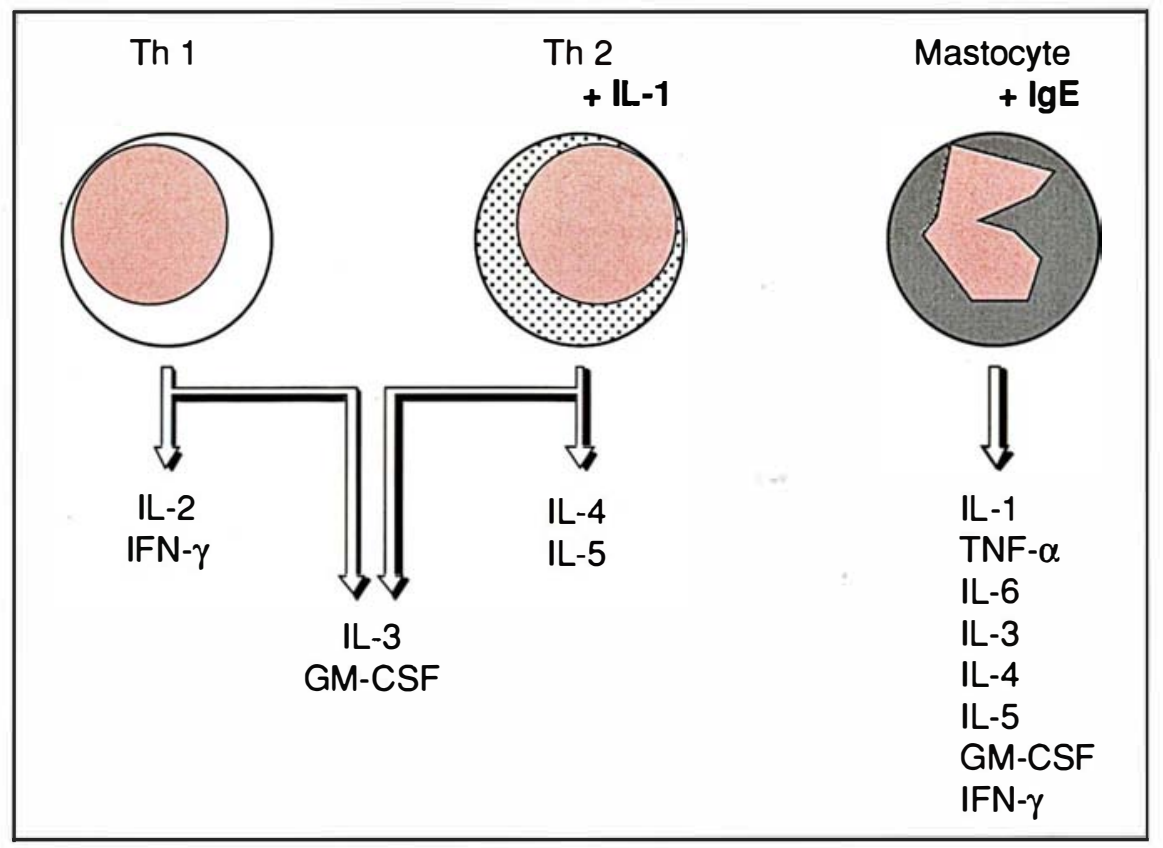

Figure 3. Compartimentation cellulaire de la production des interleukines. Chez la souris, on distingue deux cas extrêmes de cellules $T$ inductrices ou $T$ helper (Th1 et Th2) dont le profil de production de certaines interleukines est différent. Productrice d'IL-4, la cellule Th2 intervient ainsi directement dans l'induction de la réponse IgE, alors que la cellule Th1 exerce un rôle inhibiteur par l'intermédiaire de l'interféron $\gamma$. Les mastocytes activés par I'IgE peuvent également sécréter des interleukines et contribuer à la régulation de la réponse allergique.

assurée par l'IL-4 agissant sur le lymphocyte B à IgM de membrane $(\mathrm{B} \mu)$, cependant que les $I g E$-binding factors/CD23 soluble, par leur interaction avec l'IgE de membrane des lymphocytes $B_{E}$, interviennent davantage dans l'amplification de la production en cours. De manière relativement symétrique, la régulation négative de la réponse $\operatorname{IgE}$ paraît être assurée au niveau de la commutation isotypique par l'IFN- $\gamma$, cependant que le rôle présomptif des $I g E$ suppressive factors est ciblé sur les lymphocytes $B_{E}[6,17]$. Ce schéma délibérément simplifié est en réalité rendu beaucoup plus complexe, à la fois par le rôle joué par d'autres interleukines - telles les interleukines 4,5 et 6 - sur la différenciation des cellules productrices d'anticorps, mais aussi par les activités de facteur de compétence, de croissance et de différenciation des interleukines sur les cellules $\mathrm{T}$ inductrices et les lymphocytes B (figure 4).

\section{Avenir de la recherche en allergologie}

La connaissance accrue de cette machinerie cellulaire et moléculaire complexe ne doit pas occulter l'ignorance où nous restons des faits essentiels de l'induction d'une réponse allergique.

Des progrès remarquables ont cependant été réalisés récemment dans l'identification de la structure moléculaire de quelques allergènes majeurs (certains acariens et pollens) et de certains de leurs épitopes cibles d'une réponse $\operatorname{IgE}$ chez l'homme [18]. Cependant, aucune indication précise n'existe, dans les travaux actuels, permettant de répondre par des analyses structure-fonction à la plus vieille question posée par l'allergie : qu'est-ce qu'un allergène? Cette dernière interrogation, venant à la suite d'un bilan impressionnant des connaissances acquises au cours des toutes dernières années, amène 
naturellement à s'interroger sur l'avenir de la recherche en allergologie et sur les questions essentielles qui restent posées.

La première remarque - qui peut être faite sans excessif pessimisme réside dans la disproportion étonnante entre les progrès réalisés dans la connaissance des processus cellulaires et moléculaires fondamentaux, et ceux, particulièrement modestes, qui auraient pu résulter de l'application de ces connaissances dans le domaine du diagnostic, de la prévention et de la thérapie des manifestations allergiques en général. S'il est vrai que l'arsenal des outils thérapeutiques s'est enrichi de quelques innovations dignes d'intérêt, les bases conceptuelles de nos approches n'ont guère été modifiées au cours des dix

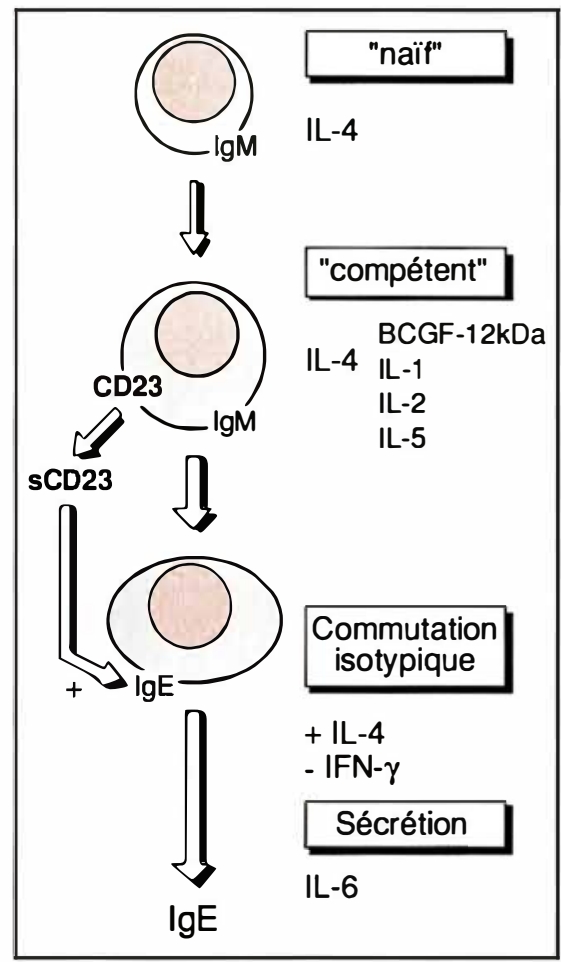

Figure 4. Rôle des interleukines et de la forme soluble du FC $R_{\mu} / C D 23$ Iymphocytaire dans la régulation de la production d'IgE. L'IL-4 est l'interleukine prédominante pour déclencher la commutation isotypique; le $\mathrm{CD} 23$ soluble (sCD23) dérivé du clivage protéolytique du $F c_{E} R_{\mu l} / C D 23$ des lymphocytes $B$ amplifie la réponse IgE en cours. BCGF : B cell growth factor; IFN : interféron; IL : interleukine. dernières années et restent confinées, souvent par empirisme, au développement d'antagonistes des médiateurs présomptivement responsables de l'expression morbide ou d'inhibiteurs de leur libération. On peut cependant rappeler que le cromoglycate et ses analogues, dont l'activité sur le mastocyte est difficilement analysable in vitro, exercent un effet inhibiteur sur la libération des médiateurs par les cellules inflammatoires $\mathrm{Fc}_{\mathrm{E}} \mathrm{R}_{\mathrm{II}}$ positives [19].

S'il est vrai que la définition précise d'objectifs prioritaires peut apparaître relativement artificielle, il n'en reste pas moins vrai que le développement de la recherche en allergologie a pour finalités essentielles la thérapeutique et même, mieux, la prévention. En ce qui concerne la thérapeutique, quelques orientations essentielles issues des progrès récents, dont la réelle signification reste à évaluer, peuvent viser au contrôle soit de l'arc effecteur, soit de l'arc inducteur de la réponse. Au niveau de l'arc effecteur, les connaissances acquises dans le domaine des récepteurs et de leur interaction avec l'IgE peuvent rendre fiable la modélisation de structures peptidiques susceptibles d'interférer efficacement avec la sensibilisation tissulaire [3, 7]. Si de nombreux arguments plaident par ailleurs en faveur de la multiplicité des médiateurs incriminés, la séquence de leur production nous est totalement inconnue et la démonstration in vivo de leur rôle reste entièrement à explorer. De telles données seraient pourtant d'une particulière importance si l'on souhaite, dépassant le contrôle de la phase aiguë de la réaction allergique, agir efficacement sur les désordres tissulaires liés aux processus inflammatoires chroniques $[13,14,20]$. Au niveau de l'arc inducteur et régulateur, s'il est encore difficile de prévoir le réel bénéfice qu'apportera en clinique humaine l'immunomanipulation par les interleukines ou leurs antagonistes, il apparaît néanmoins que cette approche reste des plus prometteuses, d'autant que, d'ores et déjà, un faisceau d'observations expérimentales in vivo indique la possibilité de modifier dans un sens favorable la réponse $\operatorname{IgE}$. Les informations que permettraient d'obtenir de telles tentatives thérapeutiques peuvent par ailleurs contribuer à une meilleure connaissance des bases immunologiques de la désensibilisation spécifique. Dans un autre domaine, si les concepts nouveaux développés grâce à la connaissance de l'hétérogénéité fonctionnelle de sous-populations lymphocytaires $\mathrm{T}$ inductrices sont d'un intérêt théorique considérable, leur réalité chez l'homme reste à démontrer. On peut cependant remarquer que l'immunothérapie spécifique, au moins dans le protocole rush de désensibilisation au venin d'hyménoptère, entraîne des modifications précoces de l'équilibre des souspopulations lymphocytaires CD4+ [21] et la libération de cytokines concourant à réduire la réactivité des cellules effectrices [22]. Il faut néanmoins souligner combien, par comparaison avec d'autres domaines de l'immunologie, la connaissance des événements cellulaires initiaux qui accompagnent l'induction et la régulation d'une réponse allergique reste plus que fragmentaire.

Longtemps freinées dans leur développement par l'absence de modèles appropriés et par la difficulté de l'abord dans les maladies humaines, l'ensemble de ces questions devrait pouvoir trouver à terme au moins quelques éléments de réponse grâce à l'utilisation des performances remarquables qu'apportent les souris immunodéficientes (scid) ou transgéniques. D'une manière plus générale encore, les outils désormais accessibles de la génétique moléculaire combinés à la transgenèse et à la mutagenèse dirigée, devraient permettre d'aborder sur des bases conceptuelles nouvelles l'analyse du "terrain" allergique ou atopique et l'identification au plan moléculaire des bases génétiques de l'allergie, dont on peut prévoir qu'elles sont plus complexes que le simple contrôle génétique de l'intensité de la production d'IgE. Ces outils devraient aussi permettre d'apprécier la contribution relative de deux classes de récepteurs dans le développement de la réaction allergique.

On aura pu mesurer, au travers de cette synthèse délibérément simplifiée de nos connaissances actuelles, combien l'allergie demeure un champ d'investigations remarquablement 


\section{RÉFÉRENCES}

13. Barnes PJ, Chung KF, Page CP. Platelet-activating factor as a mediator of allergic disease. J Allergy Clin Immunol 1988 ; 81 : 919-34.

14. Frigas E, Gleich J. The eosinophil and the pathophysiology of asthma. J Allergy Clin Immunol $1986 ; 77$ : 527-37.

15. Dessaint JP, Capron M, Capron A Immunoglobulin E-stimulated release of mediators from mononuclear phagocytes, eosinophils, and platelets. In : Metzger H, ed. Fc Receptors and the Action of Antibodies, Washington DC : Amer Soc Microbiol, $1990: 260-87$.

16. Silberstein DS, David JR. The regulation of human eosinophil function by cytokines. Immunol Today $1987 ; 8: 380-5$.

17. Pene J, Rousset F, Brière $F$, et al Interleukin 5 enhances interleukin 4-induced IgE production by normal human B cells. The role of soluble CD23 antigen. Eur $J$ Immunol 1988 ; 18 : 929-35.

18. Tovey ER, JohnsonMC, Roche AL, Cobon GS, Baldo BA. Cloning and sequencing of a cDNA expressing a recombinant house dust mite protein that binds human $\mathrm{IgE}$ and corresponds to an important low molecular weight allergen. $J$ Exp Med 1989 ; $170: 1457-62$.

19. Dessaint JP, Capron A. Interactions cellulaires dans l'allergie : les multiples cellules cibles de l'IgE. médecine/sciences 1989 ; suppl $6: 57-61$

20. Lamanske RF Jr, Kaliner M. Latephase IgE-mediated reactions. $J$ Clin Immunol $1988 ; 8: 1-20$.

21. Tilmant L, Dessaint JP, Tsicopoulos A, Tonnel AB, Capron A. Concomittant augmentation of CD4 + CD45R + suppressor inducer subset and diminution of $\mathrm{CD}^{+}+$ CDw29 + diminution during hyposensitization. Clin Exp Immunol 1989 ; 76 : 13-8.

22. Tsicopoulos A, Tonnel AB, Wallaert B, Joseph M, Ramon P, Capron A. A circulating suppressive factor of platelet cytotoxic functions after rush immunotherapy in Hymenoptera venom hypersensitivity. J Immu- contrasté. Les travaux concernant les événements cellulaires et moléculaires qui accompagnent la réponse allergique figurent certainement parmi les acquisitions les plus importantes de l'immunologie au cours des dix dernières années, cependant que, dans l'immédiat, la thérapie et a fortiori la prévention de manifestations allergiques, l'un des domaines les plus préoccupants de la pathologie humaine de par leur fréquence et leur sévérité, restent empreints d'un relatif empirisme

\section{Summary}

Allergy : present and future

Basic knowlcdge of the molecular and cellular mechanisms of allergy has greatly improved in the past few years. Several types of IgE Fc receptors have been characterized : $F_{c_{1}} R_{I}$ on mast cells and basophils, $F_{F} R_{I I}$ on subsets of mononuclear phagocytes, eosinophils, platelets, $\mathrm{B}$ and $\mathrm{T}$ cells. Through their interaction with these receptors, complexes of IgE and allergen trigger the release of proinflammatory mediators. Among these, eicosanoïdes, PAFacether, and various interleukins regulate both the production of IgE and the maturation of cffector cells. There is therefore a permanent and evolutive cross-talk among the various cells that can be triggered by IgE during the induction and the expression of the allergic response. Our therapeutic approach, in contrast, has not yet benefited of the developments in the molecular biology of the cells participating in the development of allergy.

\section{TIRÉS A PART}

A. Capron.

\section{PRIX NOBEL DE CHIMIE}

\author{
Elias T. Corey
}

Il y $a$ un an $\mathrm{m} / \mathrm{s} \quad\left(\mathrm{n}^{\circ} 8\right.$, vol. 5, p. 606) publiait une nouvelle intitulée "Les chemzymes, un nouvel outil pour la synthèse de médicaments". Elle décrivait une découverte due à Elias T. Corey, qui vient de recevoir le prix Nobel de chimie. Né en 1928, Corey fit ses études au Massachusetts Institute for Technology et travaille à Harvard University. Sa contribution essentielle, dont les fruits ont été aussi bien théoriques que pratiques, est $d$ 'avoir inventé l' analyse rétrosynthétique ", approche logique pour la synthèse de molécules complexes. Grâce à lui, ces synthèses ont perdu leur caractere empirique, remplacé par l'établissement de règles permettant de choisir, à chaque étape, la meilleure voie possible. Le commentateur de Science le compare à un grand maître du jeu d'échecs, capable de prévoir, plusieurs étapes à l'avance, quel sera l'effet de la stratégie choisie. Parmi la centaine de composés importants dont il a découvert le mode de synthèse, les plus connus sont les prostaglandines, en 1969, et plus récemment - le ginkgolide $B$, utilisé pour le traitement de l'asthme et des troubles circulatoires chez les sujets âgés.

\section{J.-C. D.}

